\title{
EFICIÊNCIA DE CONVERSÃO DA RADIAÇÃO FOTOSSINTETICAMENTE ATIVA INTERCEPTADA EM FITOMASSA DE MUDAS DE EUCALIPTO ${ }^{1}$
}

\author{
Braulio Otomar Caron², Velci Queiróz de Souza², Rômulo Trevisan², Alexandre Behling ${ }^{3}$, Denise \\ Schmidt $^{2}$, Rogério Bamberg ${ }^{3}$ e Elder Eloy ${ }^{3}$
}

\begin{abstract}
RESUMO - A produção de fitomassa é uma função da eficiência de conversão da radiação solar fotossinteticamente ativa interceptada em fotoassimilados, variando conforme as condições em que as plantas são cultivadas. Assim, este trabalho teve como objetivo quantificar a eficiência de conversão da radiação solar fotossinteticamente ativa interceptada em fitomassa - $\varepsilon b$ - de mudas de Eucalyptus grandis W. Hill ex Maiden (eucalipto) quando submetidas a diferentes tamanhos de tubetes e densidades de plantas. Para tanto, foi conduzido um experimento, em delineamento de blocos ao acaso, para estudar a produção de mudas em dois tamanhos de tubetes (pequeno e médio) e duas densidades de plantas na bandeja (alta com $100 \%$ de ocupação da bandeja e média com 50\%), em que foram determinados a radiação fotossinteticamente ativa interceptada (RFAi), o índice de área foliar e a fitomassa seca total das mudas. O volume do tubete não interferiu nos valores de eficiência, mas, sim, a densidade de plantas, pois o índice de área foliar é quem determina tal valor. A $\varepsilon b$ foi de 7,33 g. $\mathrm{MJ}^{-1}$ em tubete médio e pequeno em densidade alta e de 3,26 g.MJ-1 em tubete médio e pequeno em densidade média.
\end{abstract}

Palavras-chave: Ocupação da bandeja, Densidade de plantio e Radiação fotossinteticamente ativa.

\section{EFFICIENCY OF THE CONVERSION OF PHOTOSYNTHELICALLY ACTIVE RADIATION DRY BIOMASS IN EUCALYPTUS SEEDLINGS}

\begin{abstract}
The biomass production is function of the conversion efficiency of the photosynthetically active radiation intercepted (PARi), varying according to the conditions in which plants are cultivated. Therefore the objective of this work was to quantify the function of the efficiency of conversion of the photosynthetically active radiation intercepted (PARi) in dry biomass - $\varepsilon b$ - of seedlings of Eucalyptus grandis W. Hill ex Maiden (eucalyptus) when submitted to different sizes of tubes and densities of plants. Therefore, an experiment was conducted in randomized blocks to study seedlings production in two sizes of tubes (small and medium) and two densities of plants in the tray (high with 100\% of occupation of the tray and average with 50\% of occupation), where PARi, the index of foliar area and the total dry biomass of the seedlings were evaluated. The volume of the tube did not interfere in the efficiency values, but, the density of plants did because the index of foliar area is the one which determines such value. The åb was of $7.33 \mathrm{~g} . \mathrm{MJ}^{-1}$ for medium and small tube in high density and of $3.26 \mathrm{~g} . \mathrm{MJ}^{-1}$ for medium and small tube in medium density.
\end{abstract}

Keywords: Photosynthetically active radiation, Seedlings production of planting density.

\footnotetext{
${ }^{1}$ Recebido em 01.11.2011 aceito para publicação em 04.06.2012.

${ }^{2}$ Universidade Federal de Santa Maria no Centro de Educação Superior Norte do RS - CESNORS, Campus Frederico Westphalen. E-mail:<otomarcaron@yahoo.com.br>,<velciq@gmail.com>,<romulo_trevisan@yahoo.com.br>,<schmidtbr2000@yahoo.com.br>. ${ }^{3}$ Programa de Pós-Graduação em Mestrado em Agronomia, Agricultura e Ambiente pela Universidade Federal de Santa Maria no Centro de Educação Superior Norte do RS - CESNORS, Brasil. E-mail: <alexandre.behling@yahoo.com.br>, <rogeriobamberg@yahoo.com.br>e<eloyelder@yahoo.com.br>.
} 


\section{INTRODUÇÃO}

A implantação dos plantios florestais, em especial do gênero Eucalyptus, tem sido realizada com mudas produzidas em estufas plásticas. Todavia, o cultivo em ambiente protegido causa alterações nos elementos meteorológicos, como na radiação solar. Assim, no interior da casa de vegetação, esse elemento é um dos primeiros a serem modificados (CARON et al., 2002), sendo, consequentemente, considerado fator determinante no crescimento e desenvolvimento das mudas.

O crescimento das plantas depende do saldo de matéria seca acumulada pela fotossíntese. Em plantas sadias que possuem à disposição quantidades adequadas de água e nutrientes, a produção de fitomassa seca é governada pela radiação fotossinteticamente ativa RFA(MONTEITH, 1965, 1977). Dessa maneira, a RFA interceptada, que é convertida em fitomassa, revela a eficiência do uso da radiação - $\varepsilon b$ pela espécie (MONTEITH, 1977). Na maioria das vezes, esse mecanismo tem-se demonstrado como função linear, na qual o coeficiente angular da curva entre a RFA interceptada e a matéria seca acumulada determina a eficiência de uso da radiação para a produção de fitomassa, como constatado nos trabalhos desenvolvidos por Schöffel e Volpe (2001), Caron et al. (2002), Pereira (2002), Caron et al. (2003), Radin et al. (2003), Assunção e Martins (2008), entre outros.

Assim, uma aproximação quantitativa pode ser utilizada para determinar a relação entre produção de biomassa e radiação interceptada. Pode-se, então, aplicar tal conhecimento a diversos tópicos de pesquisa, como análise de crescimento de plantas, previsão de crescimento e desenvolvimento e estimativa do potencial de produção, sendo um modelo consistente e simplificado (MÜLLER et al., 2001; CARON et al., 2003; MÜLLER; BERGAMASCHI, 2005; ASSUNÇÃO; MARTINS, 2008).

Um dos fatores que influenciam a åb é o índice de área foliar, característica que pode ser manipulada através da densidade de plantas. Desse modo, indagações de espaçamentos têm sido conduzidas com o intuito de encurtar o período para que a cultura intercepte o máximo da radiação solar incidente por unidade de área e tempo, resultando em maior produtividade. Tais estudos têm sido conduzidos em razão de que a eficiência fotossintética é, principalmente, uma função direta da densidade de plantas, posto que o índice de área foliar é quem determina å $b$, conforme destacaram Villa Nova et al. (2003).
A densidade de plantas no viveiro tem-se delineado como importante prática de manejo para otimizar o espaço no ambiente. Além disso, exerce influências diretamente no desenvolvimento e arquitetura das plantas, apresentando, assim, padrões diferenciados em resposta aos variados espaçamentos (ATAÍDE et al., 2010), e contribui para a distribuição da área foliar no dossel e, consequentemente, na maneira como é interceptada a radiação solar (STEWART et al., 2003). Assim, uma das técnicas para aumentar a quantidade de energia captada por unidade de área e de tempo é a modificação do arranjo, a qual consiste em encurtar o tempo para que a cultura intercepte o máximo da radiação solar incidente (KUNZ et al., 2007), através do maior índice de área foliar obtido.

Dessa maneira, este trabalho teve como objetivo determinar a eficiência de conversão da radiação solar fotossinteticamente ativa interceptada em fitomassa de mudas de Eucalyptus grandis W. Hill ex Maiden submetidas a diferentes tamanhos de tubetes e densidades de plantas.

\section{MATERIAL E MÉTODOS}

O estudo foi realizado com a espécie Eucalyptus grandis W. Hill ex Maiden no período de outubro de 2008 a janeiro de 2009, em ambiente protegido com casa de vegetação do tipo Pampeana, com cobertura de polietileno de baixa densidade, com $150 \mathrm{~mm}$ de espessura. O experimento foi conduzido no viveiro do LAGRO (Laboratório de Agroclimatologia da Universidade Federal de Santa Maria, Campus CESNORS (Centro de Educação Superior Norte do Rio Grande do Sul), no Município de Frederico Westphalen - RS, sob coordenadas geográficas $27^{\circ} 23^{\prime} 46^{\prime \prime} \mathrm{S}$ e $53^{\circ} 25^{\prime} 38^{\prime \prime}$ $\mathrm{W}$ a $480 \mathrm{~m}$ de altitude.

Segundo a classificação climática de Köppen, o clima da região é Cfa. Em Iraí, município tomado como referência para dos dados de classificação climática de Frederico Westphalen, o clima é do tipo subtemperado subúmido, sendo a temperatura média anual de $18,8^{\circ} \mathrm{C}$ e temperatura média do mês mais frio de $13,3^{\circ} \mathrm{C}$ (MALUF, 2000).

O acúmulo em massa seca total de mudas de Eucalyptus grandis foi avaliado em dois tamanhos de tubetes cônicos de plástico rígido, pequeno e médio, com capacidade de 50 e $90 \mathrm{~cm}^{3}$, respectivamente, em dois níveis de densidades de plantas na bandeja, 100 
e $50 \%$, denominados densidades alta e média. A densidade alta equivaleu a 736 e 528 plantas por $\mathrm{m}^{2}$ para os tubetes pequeno e médio e para a média de 368 e 264, respectivamente.

No planejamento dos tratamentos, utilizou-se um bifatorial de 2 × 2: dois tamanhos de tubete, duas densidades de plantas na bandeja, realizados em seis repetições, sendo o experimento conduzido em delineamento experimental de blocos ao acaso. Em cada bloco foram contempladas quatro unidades experimentais, distribuindo-se ao acaso a combinação tamanho de tubete + densidades de plantas na bandeja. Cada unidade experimental foi composta por 66 e 48 plantas, respectivamente para 100 e $50 \%$ de ocupação na bandeja, sendo as 16 centrais avaliadas.

A produção das mudas foi realizada a partir de sementes oriundas de povoamentos clonais da empresa KLABIN® de Telêmaco Borba - PR. Na semeadura, realizada em 24 de agosto de 2008, colocaram-se 10 sementes por tubete preenchidos com substrato comercial (Tecnomax®); aos 15 dias após a germinação, foi efetuado um raleio das plântulas, deixando-se somente aquela melhor e mais central. Durante o experimento, fizeram-se regas diárias de forma manual, mantendo sempre a capacidade de campo do substrato.

A partir dos 50 dias após a germinação, realizaram-se sete avaliações de fitomassa, com intervalos de 15 dias, quando duas mudas por tratamento e repetição foram amostradas. No total, 336 mudas foram avaliadas.

A produção de fitomassa seca foi estuda com base no modelo proposto por Monteith (1977), em que esta é uma variável dependente da quantidade de radiação fotossinteticamente ativa interceptada e multiplicada pela eficiência de conversão $(\varepsilon b)$ dessa radiação em fitomassa seca produzida. Dessa forma, a $\varepsilon b$ pode ser calculada por meio da relação entre a produção média de fitomassa seca acumulada e a radiação fotossinteticamente ativa interceptada envolvida na produção de fitomassa, conforme a seguinte expressão:

$$
\mathrm{PFS}=\varepsilon b * \mathrm{RFAi}
$$

em que PFS é a produção de fitomassa seca $\left(\mathrm{g} \cdot \mathrm{m}^{-2}\right)$; RFAi é a radiação solar fotossinteticamente ativa interceptada $\left(\mathrm{MJ}^{-\mathrm{m}^{-2}}\right)$ e $\varepsilon b$ é a eficiência de conversão da radiação RFAi em fitomassa seca produzida $\left(\mathrm{g} \cdot \mathrm{MJ}^{-1}\right)$.
A radiação fotossinteticamente ativa interceptada foi determinada com base no modelo proposto por VarletGrancher et al. (1989):

$$
\mathrm{RFAi}=0,95 *\left(\mathrm{RFA}_{\text {inc }}\right) *\left(1-\mathrm{e}^{(-\mathrm{k} * \mathrm{IAF})}\right)
$$

em que RFAi é a radiação fotossinteticamente ativa interceptada (MJ.m ${ }^{-2}$ ), K uma constante adimensional que depende das propriedades óticas das folhas e da geometria do dossel vegetal $(0,8)$, IAF o índice de área foliar (adimensional) e RFAinc a radiação fotossinteticamente ativa incidente $\left(\mathrm{MJ} \cdot \mathrm{m}^{-2}\right)$.

O índice de área foliar foi determinado a partir da área foliar total de cada planta e da área de bandeja explorada por esta (densidade de mudas), calculado através da equação:

$$
\mathrm{IAF}=\frac{\mathrm{AF}}{\mathrm{AEB}}
$$

em que IAF é o índice de área foliar (adimensional), $\mathrm{AF}$ a área foliar total da planta em $\mathrm{m}^{2} \mathrm{e} \mathrm{AEB}$ a área de bandeja explorada pela planta $\mathrm{em}^{2}$.

A área foliar foi obtida através do uso do seguinte modelo, obtido nas condições do experimento:

$$
\begin{aligned}
& \mathrm{AF}=\left[\left(\mathrm{c}_{1} * 1_{1}\right) * 0,7226\right] * \mathrm{nfp}+\left[\left(\mathrm{c}_{2} * 1_{2}\right) *\right. \\
& 0,7226] * \mathrm{nfm}+\left[\left(\mathrm{c}_{3} * 1_{3}\right) * 0,7226\right] * \mathrm{nfg}
\end{aligned}
$$

em que $\mathrm{AF}=$ área foliar em $\mathrm{cm}^{2} ; \mathrm{c}_{1}=$ comprimento mediano da folha de tamanho pequeno, $\mathrm{em} \mathrm{cm} ; \mathrm{c}_{2}=$ comprimento mediano da folha de tamanho médio, em cm; $\mathrm{c}_{3}=$ comprimento mediano da folha de tamanho grande, em cm; $1_{1}=$ largura mediana da folha de tamanho pequeno, em cm; $1_{2}=$ largura mediana da folha de tamanho médio, em cm; $1_{3}=$ largura mediana da folha de tamanho grande, em $\mathrm{cm} ; \mathrm{nfp}=$ número total de folhas pequenas (até $2,5 \mathrm{~cm}$ de comprimento); $\mathrm{nfm}=$ número total de folhas médias (de 2,6 a 5 cm de comprimento); nfg = número total de folhas grandes (maior que $5 \mathrm{~cm}$ de comprimento); e 0,7226 = fator de correção do retângulo (comprimento $\mathrm{x}$ largura da folha).

A radiação solar global dentro da estufa foi estimada considerando a transmissividade da cobertura plástica de $53 \%$ da radiação solar global incidente sobre a cobertura da estufa, que foi medida no início da condução do experimento, utilizando-se um piranômetro (LI200X, Li Cor). Os valores de radiação solar global foram obtidos na Estação Climatológica do Instituto Nacional de

Revista Árvore, Viçosa-MG, v.36, n.5, p.833-842, 2012 
Meteorologia (INMET), situada a cerca de $150 \mathrm{~m}$ do experimento. Dessa maneira, a Rge foi calculada com base na seguinte expressão:

$$
\text { Rge }=0,53 * \mathrm{RG}
$$

em que Rge é a radiação solar global dentro da estufa e $\mathrm{Rg}$, a radiação solar global incidente sobre a cobertura da estufa.

A radiação fotossinteticamente ativa incidente foi estimada considerando-se como $45 \%$ da radiação solar global. Essa fração representa o valor médio daqueles encontrados para o Rio Grande do Sul por Assis e Mendez (1989) e Pandolfo (1995). A estimativa da radiação fotossinteticamente ativa acumulada foi realizada com base em Monteith (1977) e Varlet-Grancher et al. (1989).

A fitomassa seca total das plantas foi determinada a partir da soma dos compartimentos: folha, caule e raiz (BENINCASA, 2003). Cada compartimento foi posto em sacos de papel devidamente identificados, sendo estes levados à estufa de circulação forçada na temperatura de $60^{\circ} \mathrm{C}$ até atingir peso constante (SILVA et al., 2007; RESENDE et al., 2011). Posteriormente, pesou-se o material em balança de precisão, para obtenção do peso da matéria seca.

A análise de covariância (ANACOVA) foi aplicada para verificar a necessidade do uso de funções independentes entre a radiação fotossinteticamente ativa interceptada e o acúmulo de fitomassa seca total nos diferentes tratamentos estudados, determinando, assim, se existem diferenças entre eles. Primeiramente, testou-se a diferença de inclinação (paralelismo). Não existindo evidência desse fator, testou-se, também, a hipótese da não diferença de níveis (coincidência). Se a diferença não for significativa para inclinação e níveis, uma regressão única pode ser ajustada. Primeiramente, aplicou-se a ANACOVA entre todos os tratamentos e, posteriormente, entre combinações de tratamentos. Para tanto, foi utilizado o método de Snedecor, sendo a inclinação e nível das restas verificadas através do valor F, para $1 \%$ de erro.

\section{RESULTADOS}

O fluxo de radiação solar global foi, em média, de $27,46 \mathrm{MJ} . \mathrm{m}^{-2} \cdot \mathrm{dia}{ }^{-1}$, com variação de 2,12 a 44,65 MJ.m ${ }^{-2} \cdot$ dia $^{-1}$ e dentro da estufa, em média de 14,55 MJ.m² ${ }^{-2} \cdot$ dia $^{-1}$, com variação de 1,12 a 23,67 MJ.m². dia $^{-1}$.
O crescimento em fitomassa seca dos tratamentos apresentou relação linear positiva com a radiação fotossinteticamente ativa interceptada acumulada, com altos coeficientes de correlação e padrão de resposta principalmente dependente da densidade de mudas. O mesmo foi observado com o índice de área foliar, conforme demonstrado na Figura 1.

O índice de área foliar sempre foi maior nas densidades altas, embora a área foliar não tenha seguido a mesma tendência, pois os maiores valores foram observados nos tubetes de tamanho médio, independente da densidade (Figura 1).

Quando foram observados os coeficientes angulares obtidos, que expressam o valor de eficiência de utilização de energia solar pelas mudas, constatou-se que, ao aumentar a densidades de plantas, maior foi a eficiência no uso da radiação. A eficiência de conversão - $\varepsilon b$ de fitomassa seca total de mudas de eucalipto em relação à quantidade de radiação solar fotossinteticamente ativa interceptada acumulada foi de 7,76 g. $\mathrm{MJ}^{-1}$ em tubete médio em densidade alta, 6,88 g.MJ-1 em tubete pequeno em densidade alta, 4,17 g. $\mathrm{MJ}^{-1}$ em tubete médio em densidade média e 3,7 g. $\mathrm{MJ}^{-1} \mathrm{em}$ tubete médio em densidade média (Figura 1E).

Analisando o fator tamanho de tubete dentro de cada densidade de muda, percebe-se, de acordo com a Figura 1E, que, embora cada densidade tenha apresentado diferentes valores de $\varepsilon(b)$, ambas tiveram a mesma tendência de acréscimo e os valores $\varepsilon(b)$ de cada densidade de mudas foram semelhantes.

A análise de covariância (ANACOVA) aplicada a regressões independentes em todos os tratamentos (tubete pequeno em densidade média*, tubete médio em densidade média*, tubete pequeno em densidade alta* e tubete médio em densidade alta) evidenciou existir diferença significativa entre os valores de eficiência, indicando que as regressões apresentam inclinações e níveis diferentes com a sua variação. No entanto, quando verificadas as regressões independentes para diferentes tamanhos de tubetes submetidos à mesma densidade, tubete pequeno em densidade média*, tubete médio em densidade média e tubete pequeno em densidade alta* e tubete médio em densidade alta, a análise não mostrou evidências de existir diferença significativa, indicando que as inclinações e níveis não variaram com o tamanho do tubete, mas, sim, com a densidade de plantas (Tabela 1).

Revista Árvore, Viçosa-MG, v.36, n.5, p.833-842, 2012 
TPDA: tubete pequeno em arranjo de densidade alta; TPDM: tubete pequeno em arranjo de densidade média; TMDA: tubete médio em arranjo de densidade alta; TMDM: tubete médio em arranjo de densidade média; TPDA + TMDA = tubete pequeno e médio em arranjo de densidade alta; TPDM + TMDM = tubete pequeno e médio em arranjo de densidade média; sendo densidade alta com $100 \%$ de ocupação da bandeja e densidade média com $50 \%$ de ocupação da bandeja, sendo o volume do tubete pequeno de $50 \mathrm{~cm}^{3}$ e tubete médio de $90 \mathrm{~cm}^{3}$.
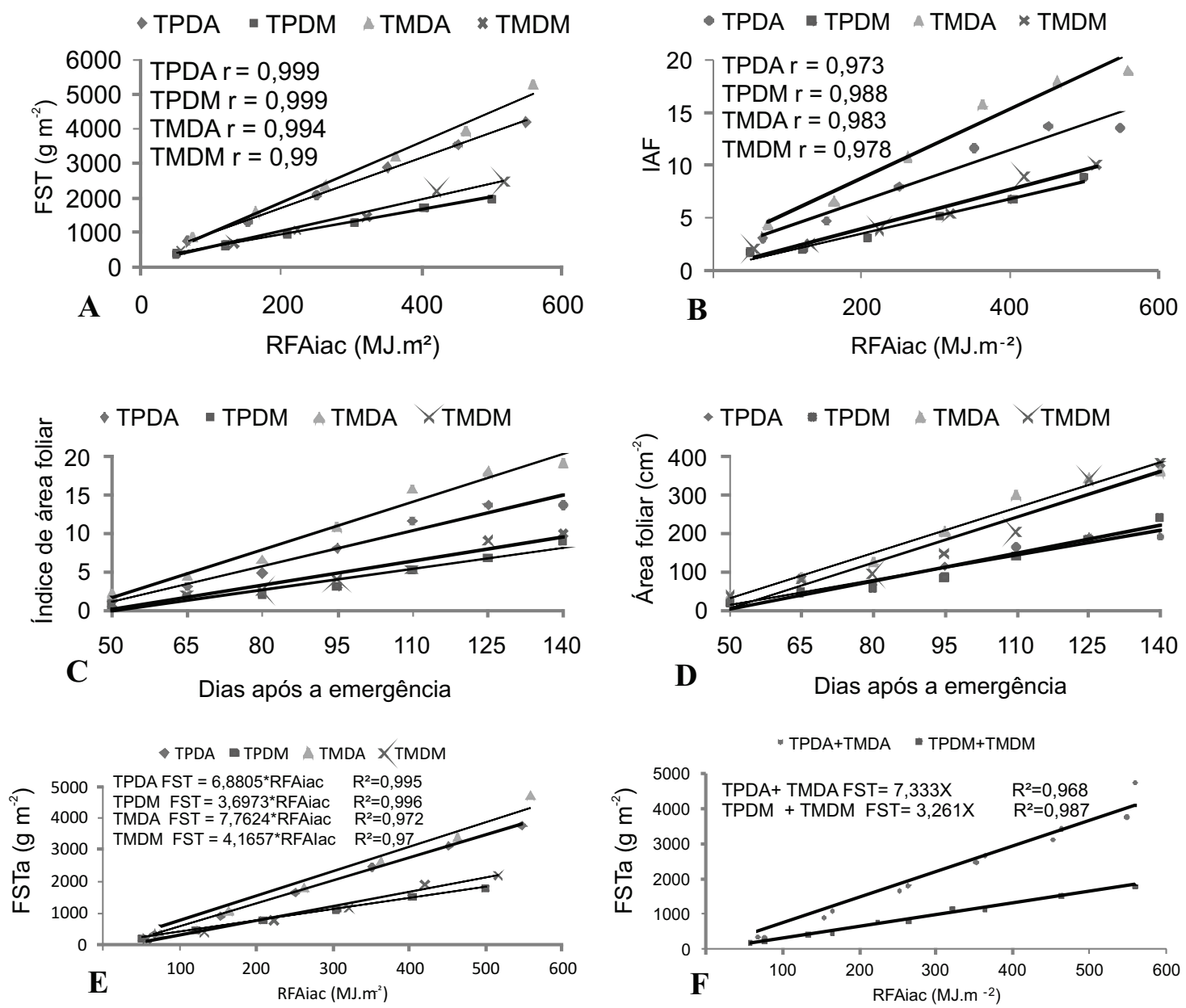

Figura 1 - Correlação de Pearson entre a fitomassa seca total - FST (A) de mudas de Eucalyptus grandis W. Hill ex Maiden e o índice de área foliar - IAF (B) com a radiação fotossinteticamente ativa interceptada acumulada (RFAiac), crescimento do índice de área foliar (C) e área foliar (D); relação entre RFAiac e a produção de fitomassa seca acumulada (FSTa) (E); e relação RFAiac e a produção de fitomassa seca acumulada (FSTa) (F) após verificar a igualdade de nível e declive entre o mesmo tamanho de tubete numa mesma densidade através da análise de covariância.

Figure 1 - Correlation of Pearson between the dry biomass - FST $(A)$ in seedlings of Eucalyptus grandis W. Hill ex Maiden and the index of foliar area - IAF $(B)$ with the photosynthetically active radiation intercepted (PARiac); growth of the foliar area index $(C)$ and foliar area $(D)$ in function of the days after germination; relationship among the PARiac and the production of accumulated dry biomass (FSTa) (E); Relationship among the PARiac and the production of accumulated dry biomass $(F S T a)(F)$ after verifying the level equality and slope among the same tube size in a same density through the covariance analysis.

Os dois grupos obtidos revelaram que a $\varepsilon(b)$ pode explicar mais que $96 \%$ da produção de fitomassa e com erro-padrão da estimava menor que $12 \%$ em ambos os casos. Ainda, os valores de erro-padrão do coeficiente angular foram baixos, sendo de 0,21 no grupo tubete pequeno em densidade alta*, tubete médio em densidade alta e de 0,11 para tubete pequeno em densidade média* e tubete médio em densidade média. 
Tabela 1 - Análise de covariância entre retas de eficiência de conversão de radiação fotossinteticamente ativa interceptada e acumulada em fitomassa seca de mudas de Eucalyptus grandis W. Hill ex Maiden submetidas a diferentes tamanhos de tubetes e densidades de plantas no Município de Frederico Westphalen, RS.

Table 1 - Covariance analysis among the straight lines of conversion efficiency of the photosynthetically active radiation intercepted and accumulated in dry biomass of seedlings of Eucalyptus grandis W. Hill ex Maiden submitted to different tubes size and densities of plants in Frederico Westphalen, $R S$

\begin{tabular}{|c|c|c|c|}
\hline Fator de variação & $\begin{array}{l}\text { Grau de liberdade } \\
\text { Para todo o grupo }\end{array}$ & Quadrado médio & Igualdade do parâmetro \\
\hline TPDM*TPDA*TMDM*TMDA & 16 & 13880,92486 & \\
\hline Inclinação & 3 & $888295,957604749 * *$ & Não \\
\hline Nível & 3 & $1718122,59572804 * *$ & Não \\
\hline \multicolumn{4}{|l|}{ Para TPDM*TMDM } \\
\hline TPDM*TMDM & 8 & 7902,314321 & \\
\hline Inclinação & 1 & $80087,8055145191^{\mathrm{ns}}$ & Sim \\
\hline Nível & 1 & $15961,1599103761^{\mathrm{ns}}$ & Sim \\
\hline \multicolumn{4}{|l|}{ Para TPDA*TMDA } \\
\hline TPDA*TPMA & 8 & 19859,53539 & \\
\hline Inclinação & 1 & $189002,055008927^{\mathrm{ns}}$ & Sim \\
\hline Nível & 1 & $132813,980795924^{\mathrm{ns}}$ & Sim \\
\hline
\end{tabular}

Em que $* *=$ significante a $1 \%$ de probabilidade de confiança; ns = não significante a $1 \%$ de probabilidade de confiança; TPDA = tubete pequeno em arranjo de densidade alta; TPDM = tubete pequeno em arranjo de densidade média; TMDA = tubete médio em arranjo de densidade alta; e TMDM = tubete médio em arranjo de densidade média.

\section{DISCUSSÃO}

A disponibilidade de radiação solar global incidente no ambiente estufa foi menor devido à transmissividade do plástico, no entanto tal diminuição pode ser compensada em parte pelo aumento da fração da radiação difusa, que tem sua importância por ser multidirecional e penetrar melhor no interior do dossel vegetativo, conforme destacaram Buriol et al. (1995). Diferentes autores encontraram valores maiores de radiação difusa dentro de estufas de plástico do que no ambiente externo, tal como Farias et al. (1993), que verificaram que a radiação difusa externamente foi inferior, em média, a $65 \%$ daquela observada no interior da estufa.

A densidade de plantas influenciou o incremento do índice de área foliar, pois afeta a distribuição da área foliar no dossel das plantas e, principalmente, o índice de área foliar. Logo, o maior acúmulo em matéria seca obtida em função da radiação fotossinteticamente ativa interceptada e acumulada está estreitamente ligado ao índice de área foliar, pois, à medida que esse se eleva, maior será a quantidade de radiação fotossinteticamente ativa interceptada e, consequentemente, o acúmulo em fitomassa, o que também foi observado por Stewart et al. (2003).

Ao elevar a densidade de mudas na bandeja, a fitomassa acumulada por mudas clonais de eucalipto também aumentou, como constataram Ataíde et al. (2010), assim como Távora et al. (2002), que trabalhando com amendoim encontraram taxas de rendimentos crescentes com a densidade de plantio. Essas situações evidenciam que o arranjo de plantas é importante prática de manejo, tendo em vista a interação com a interceptação de radiação solar, que é um dos principais fatores determinantes do crescimento e desenvolvimento de dada cultura. $\mathrm{O}$ efeito da luz no crescimento das mudas pode ser constatado em diversos trabalhos, como de Alvarenga et al. (2003), Mazuchowski et al. (2007), Silva et al. (2007), Dantas et al. (2009) e Rezende et al. (2011).

A eficiência na interceptação da radiação fotossinteticamente ativa está diretamente relacionada ao índice de área foliar da cultura, pois este fator influi no desenvolvimento, duração da área foliar e no potencial de produção da fitomassa (MAYERS et al., 1991), visto que seu aumento proporciona incremento na interceptação de radiação.

Os valores de eficiência de conversão da radiação fotossinteticamente ativa em fitomassa de mudas de eucalipto foram superiores aos observados em cultivos, dentro de estufa, de melão por Caron et al. (2002) de 2,21 g. $\mathrm{MJ}^{-1}$ e de alface por Caron et al. (2003) de $1,28 \mathrm{~g} \cdot \mathrm{MJ}^{-1}$ no cultivo de verão e $1,8 \mathrm{~g} \cdot \mathrm{MJ}^{-1}$ no de primavera. Para cultivos fora de estufa, de 1,44 g.MJ ${ }^{-1}$ para o amendoim por Assunção e Martins (2008), de 
1,52 a 2,99 MJ.m² ${ }^{-2}$ dependendo do genótipo em ervilha por Lecoeur e Ney (2003) e de 1,73 para o arroz por Steinmetz e Siqueira (2001).

Na literatura nacional, não foram encontrados trabalhos com mudas de espécies florestais referentes a este estudo, o que permite admitir a importância de intensificar pesquisas que relacionam o uso da luz solar, pois, como se sabe, o crescimento e desenvolvimento de qualquer vegetal dependem da taxa fotossintética e da eficiência de conversão da energia disponível em massa seca de planta.

Os maiores valores de eficiência de conversão observada nos tratamentos com densidade alta de plantas está relacionada à ocupação mais rápida da área foliar do espaço entre as mudas, que pode ser constatado através do elevado índice de área foliar durante todo o período. Ao aumentar o índice de área foliar, eleva-se, por conseguinte, a superfície de absorção de radiação e, também, do acúmulo de radiação fotossinteticamente ativa; proporcionalmente, a produção de fitomassa também é maior, pois essa é uma função linear, como constatado, do acúmulo de radiação fotossinteticamente ativa interceptada.

Verificou-se, assim, que o volume do tubete não interfere nos valores de eficiência, mas sim, como constatado e também observado por Villa Nova et al. (2003), que a eficiência fotossintética é função direta da densidade de plantas, pois o índice de área foliar é quem determina tal valor. Desse modo, a eficiência de conversão - $\varepsilon b$ de fitomassa seca total de mudas de eucalipto em relação à quantidade de radiação solar fotossinteticamente ativa interceptada acumulada foi de 7,33 g. $\mathrm{MJ}^{-1}$ em tubete médio e pequeno em densidade alta e de 3,26 em tubete médio e pequeno em densidade média (Figura 1F).

Dessa maneira, pode-se inferir que, conforme os resultados da Figura 1F, na maior densidade de plantas - alta, obteve-se maior a eficiência de interceptação da RFA. E isso ocorre devido à ocupação mais rápida da área foliar do espaço entre as mudas, durante o período de crescimento, como também observado por Kunz et al. (2007), o que permite dentro de um mesmo período maior interceptação de RFA.

De modo geral, os valores de eficiência de uso da radiação são maiores em cultivos dentro de estufas plásticas, em comparação com ambiente natural, e isso é explicado pelo aumento da radiação difusa nesse ambiente, conforme citaram Hammer e Vanderlip (1989). $\mathrm{O}$ aumento da radiação difusa promove maior uniformidade da radiação no interior do dossel, fazendo que as folhas inferiores aumentem a interceptação de radiação e também o seu uso. Além disso, folhas sujeitas a altas intensidades de radiação solar podem levá-las à saturação luminosa, diminuindo a eficiência no uso da radiação (JIANG et al., 2004). A maior eficiência de uso da radiação na densidade alta de plantas na bandeja também pode ser explicada por causa do aumento da contribuição relativa das folhas sombreadas para o acúmulo de fitomassa das mudas de eucalipto, o que também foi constatado em tomateiro por Radin et al. (2003).

No cultivo de mudas, o suprimento de água e nutrientes não se apresentam como fatores limitantes, devido à possibilidade de controle desses elementos. Assim, a produção de fitomassa é controlada pela radiação solar, o que foi observado e também proposto por Monteith (1965). Dessa maneira, o crescimento de mudas de eucalipto dependerá da quantidade de radiação fotossinteticamente ativa interceptada e da eficiência de utilização dessa energia no processo fotossintético para produção de fitomassa, bem como da densidade de plantas na bandeja.

A eficiência da conversão do cultivo das mudas em tubetes médio e pequeno sob densidade alta equivaleu a aproximadamente $44 \%$ maior quando comparada com a dos tubetes submetidos a densidade média. Dessa forma, a maior eficiência de conversão obtida nas densidades altas favorece o maior acúmulo de fitomassa, diminuindo o tempo da produção de uma mesma quantidade de mudas. Já Purcell et al. (2002) avaliaram a eficiência do uso de radiação e produção de biomassa de soja, em diferentes populações de plantas, e observaram a interceptação de radiação aumentou até a densidade de aproximadamente 50 plantas por $\mathrm{m}^{2}$ e que a eficiência do uso de radiação decresceu com o aumento da população de plantas, devido à senescência de folhas inferiores, resultados adversos aos observados nas mudas de eucalipto.

A modelagem da eficiência pela qual a radiação solar incidente é utilizada no acúmulo de fitomassa de mudas de eucalipto demonstra-se ser consistente em modelos potenciais e apropriada para a análise do crescimento de mudas, que também já foi indicada por Müller e Bergamaschi (2005) para outros cultivos. Essa

Revista Árvore, Viçosa-MG, v.36, n.5, p.833-842, 2012 
situação também é evidenciada através das excelentes estatísticas de ajuste das relações obtidas entre $\varepsilon(b)$ e a produção de fitomassa, em que tal relação pode explicar mais que $96 \%$ da produção de fitomassa e com erro-padrão da estimava menor que $12 \%$ em ambos os casos. No entanto, critérios técnicos como o espaço disponível no viveiro, o custo com tubetes (GOMES et al., 2003), substratos e, sobre tudo, o manejo das mudas, também devem ser levados em consideração na escolha de uso de um tamanho de tubete em dada densidade.

Para produzir mudas em menor espaço de tempo, deve-se utilizar a densidade alta, tendo em vista a maior eficiência em converter radiação fotossinteticamente ativa em fitomassa. No entanto, nessa condição os cuidados de estiolamento das mudas e com doenças devem ser reforçados, pois a sobrevivência, o estabelecimento, a frequência dos tratos culturais e o crescimento inicial das florestas estão estreitamente relacionados com a qualidade das mudas (FONSECA, 2000). Entretanto, há a possibilidade de produzir mais mudas num mesmo espaço e período de tempo.

\section{CONCLUSÕES}

Os resultados indicaram que:

O volume do tubete não interfere nos valores de eficiência, mas, sim, na densidade de plantas, pois o índice de área foliar é que determina tal valor.

A eficiência de conversão de radiação solar fotossinteticamente ativa - $\varepsilon(b)$ é de $7,33 \mathrm{~g} \cdot \mathrm{MJ}^{-1}$ para tubetes médio e pequeno em densidade alta e 3,26 para tubete médio e pequeno em densidade média.

Para um mesmo valor de radiação fotossinteticamente ativa interceptada, obtém-se maior eficiência de uso da radiação no acúmulo em matéria seca quando as mudas de E. grandis são cultivadas em densidade alta, resultando em menor tempo do cultivo para um mesmo número de plantas.

Com este trabalho, um novo campo de pesquisa na área florestal é apresentado, evidenciando que para futuros estudos de $\varepsilon(b)$ a densidade de mudas deve ser priorizada no lugar do tamanho de tubete e, ainda, apresenta um modelo muito atrativo para estudar o efeito de diversos tratamentos no crescimento de mudas.

\section{AGRADECIMENTOS}

Ao CNPq, pelo apoio financeiro.

\section{REFERÊNCIAS}

ALVARENGA, A. A. et al. Effects of different light levels on the initial growth and photosynthesis of Croton urucurana Baill. in southeastern Brazil. Revista Árvore, v.27, n.1, p.53-57, 2003.

ASSIS, F. N.; MENDEZ, M. E. G. Relação entre radiação fotossinteticamente ativa e radiação global. Pesquisa Agropecuária Brasileira, v.2, n.7, p.797-800, 1989.

ASSUNÇÃO, I. C. A.; MARTINS, D. Eficiência de uso da radiação fotossinteticamente ativa pela cultura do amendoim (Arachis hypogaea L). Energia na Agricultura, v. 23, n.4, p.34-43, 2008.

ATAÍDE, M. G. et al. Efeito da densidade na bandeja sobre o crescimento de mudas de eucalipto. Revista Trópica, v.4, n.2, p.21, 2010.

BENINCASA, M. M. P. Análise de crescimento de plantas (noções básicas). Jaboticabal: FCAVUNESP, 2003. 42p.

BURIOL. G. A. et al. Transmissividade da radiação solar do polietileno de baixa densidade utilizado em estufa. Ciência Rural, v.25, n.1, p.1-4, 1995.

CARON, B. A. et al. Eficiência de conversão da radiação solar fotossinteticamente ativa e distribuição de fitomassa produzida no meloeiro cultivado em estufa plástica na primavera.

Revista Brasileira de

Agrometeorologia, v.10, n.2, p.215-219, 2002.

CARON, B. et al. Eficiência de conversão da radiação solar fotossinteticamente ativa interceptada em fitomassa de alface. Revista Brasileira de Agrometeorologia, v.11, n.2, p.261-268, 2003.

DANTAS, B. F. et al. Taxas de crescimento de mudas de catingueira submetidas a diferentes substratos e sombreamentos. Revista Árvore, v.33, n.3, p.413-423, 2009. 
FARIAS, J. R. B.; BERGAMASCHI, H.; MARTINS, S. R. Efeito da cobertura plástica sobre a radiação solar. Revista Brasileira de

Agrometeorologia, v.1, n.1, p.31-36, 1993.

FONSECA, E. P. Padrão de qualidade de mudas de Trema micrantha (L.) Blume., Cedrela fissilis Vell. e Aspidosperma polyneuron Müll. Arg. produzidas sob diferentes períodos de

sombreamento. 2000. 113f. Tese (Doutorado em Agronomia) - Universidade Estadual

Paulista, São Paulo, 2000.

GOMES, J. M. et al. Crescimento de mudas de Eucaplyptus gandis em diferentes tamanhos de tubetes e fertilização N-P-K. Revista Árvore, v.27, n.2, p.113-127, 2003.

HAMMER, G. L.; VANDERLIP, R. L. Genotypebyenvironment interaction in grain sorghum - I: effects of temperature on radiation use efficiency. Crop Science, v.29, n.2, p.370-376, 1989.

JIANG, A. C. D. et al. Leaf orientation, photorespiration and xanthophyll cycle protect young soybean leaves against high irradiance in field. Environmental and Experimental Botany, v.30, n.2, p.1-10, 2004.

KUNZ, J. H. et al. Uso da radiação solar pelo milho sob diferentes preparos do solo, espaçamento e disponibilidade hídrica.

Pesquisa Agropecuária Brasileira, v.42, n.11, p.1511-1520, 2007.

LECOEUR, J.; NEY, B. Change with time in potential radiation-use efficiency in field pea. European Journal of Agronomy, v.19, n.1, p.91-105, 2003.

MALUF, J. R. T. Nova classificação climática do Estado do Rio Grande do Sul. Revista Brasileira de Agrometeorologia, v.8, n.1, p.141-150, 2000.

MAYERS, J. D.; LAWN, R. J.; BYTH, D. E. Agronomic studies on soybean (Glycine max (L.) Merrill) in the dry seasons of the tropics. II. Interaction of sowing date and sowing density. Australian Journal of Agricultural Research, v.42, n.7, p.1093-1107, 1991.
MAZUCHOWSKI, J. Z.; SILVA, E. T.; MACCARI, J. A. Efeito da luminosidade e da adição de nitrogênio no crescimento de plantas de Ilex paraguariensis St. Hil. Revista Árvore, v.31, n.4, p.619-627, 2007.

MONTEITH, J. L. Climate and the efficiency of crop production en Britain. Proceedings of the Royal Society of London, v.281, n.980, p.277-294, 1977.

MONTEITH, J. L. Light distribution and photosynthesis in field crops. Annals of Botany, v.29, p.17- 37, 1965.

MÜLLER, A. G.; BERGAMASCHI, H.; SILVA, M. I. G. Eficiências de interceptação, absorção e de uso da radiação fotossinteticamente ativa pelo milho (Zea mays L.), em diferentes disponibilidades hídricas. In: CONGRESSO BRASILEIRO DE AGROMETEOROLOGIA, 12.; REUNIÃO LATINOAMERICANADE AGROMETEOROLOGIA, 3., 2001, Fortaleza. Anais... Fortaleza: Sociedade Brasileira de Agrometeorologia, 2001. p.565-566. CD ROM.

MÜLLER. A. G.; BERGAMASCHI, H. Eficiências de interceptação, absorção e uso da radiação fotossinteticamente ativa pelo milho (Zea mays L.), em diferentes disponibilidades hídricas e verificação do modelo energético de estimativa da massa seca acumulada. Revista

Brasileira de Agrometeorologia, v.13, n.1, p.27-33, 2005.

PANDOLFO, C. Parâmetros básicos para uso na modelagem do rendimento de matéria seca de alfafa (Medicago sativa L.). 1995. 128f. Dissertação (Mestrado em Agronomia) - Universidade Federal do Rio Grande do Sul, Porto Alegre, 1995.

PEREIRA, C. R. Análise do crescimento e desenvolvimento da cultura de soja sob diferentes condições ambientais. 2002. 282f. Tese (Doutorado em Engenharia Agrícola)-Universidade Federal de Viçosa, Viçosa, MG, 2002.

PURCELL, L. C. et al. Radiation use efficiency and biomass production in soybean at different plant population densities. Crop Science, v.42, p.172-177, 2002.

Revista Árvore, Viçosa-MG, v.36, n.5, p.833-842, 2012 
RADIN, B. et al. Eficiência de uso da radiação fotossinteticamente ativa pela cultura do tomateiro em diferentes ambientes. Pesquisa Agropecuária Brasileira, v. 38, n.9, p.1017-1023, 2003.

RESENDE, S. V. et al. Influência da luz e substrato na germinação e desenvolvimento inicial de duas espécies de Calliandra Benth: (Mimosoideae - Leguminosae) endêmicas da Chapada Diamantina, Bahia. Revista Árvore, v.35, n.1, p.107-117, 2011.

SCHÖFFEL, E. R.; VOLPE, C. A. Eficiência de conversão da radiação fotossinteticamente ativa interceptada pela soja para a produção de fitomassa. Revista Brasileira de

Agrometeorologia, v.9, n.2, p.241-249, 2001.

SILVA, B. M. S. et al. Efeito da luz no crescimento de mudas de Hymenaea parvifolia Huber.

Revista Árvore, v.31, n.6, p.1019-1026, 2007.

STEINMETZ, S.; SIQUEIRA, O. J. W. Eficiência de conversão em biomassa da radiação solar interceptada nas distintas fases do ciclo de três tipos de planta de arroz irrigado. In:
CONGRESSO BRASILEIRO DE

AGROMETEOROLOGIA, 12.; REUNIÃO

LATINOAMERICANADE

AGROMETEOROLOGIA, 3., 2001. Fortaleza, CE,

Anais... Fortaleza : Sociedade Brasileira de

Agrometeorologia, 2001. p.691-692.

STEWART, D. W. et al. Canopy structure, light interception and photosynthesis in maize.

Agronomy Journal, v.95, p.1465-1474, 2003.

TÁVORA, F. J. F. et al. Peanut response to plant densities and planting patterns: light interception, growth analysis and yield. Revista Ciência Agronômica, v.33, n.2, p.5-12, 2002.

VARLET-GRANCHER, C. et al. Mise au point: rayonnement solaire absorbé ou intercepté par um couvert végétal. Agronomie, v.9, n.5, p.419-439, 1989.

VILLA NOVA, N. A.; MOREIRA, P. R.; PEREIRA, A. B. Eficiência de captura de energia solar por um dossel de Eucalyptus pellita F. Muell sob várias densidades de plantio. Revista Brasileira de Agrometeorologia, v.11, n.2, p.63-68, 2003. 